\title{
Correction to: An overview and evaluation of citation recommendation models
}

\author{
Zafar Ali ${ }^{1}$ D Irfan Ullah² $\cdot$ Amin Ul Haq ${ }^{3} \cdot$ Asim Ullah Jan $^{5} \cdot \mathrm{Khan} \mathrm{Muhammad}^{4}$ \\ Published online: 7 June 2021 \\ (c) Akadémiai Kiadó, Budapest, Hungary 2021
}

\section{Correction to: Scientometrics (2021) 126:4083-4119 https://doi.org/10.1007/s11192-021-03909-y}

In the original publication of the article the third author name and their corresponding affiliation was incorrectly published. This has been corrected with this Correction.

The original article can be found online at https://doi.org/10.1007/s11192-021-03909-y.

Zafar Ali

zafarali@seu.edu.cn

$\triangle$ Khan Muhammad

khan.muhammad@ieee.org

Irfan Ullah

irfan@sbbu.edu.pk

Amin Ul Haq

khan.amin50@yahoo.com

Asim Ullah Jan

asim.ibms@gmail.com

1 School of Computer Science and Engineering, Southeast University, Nanjing, China

2 Department of Computer Science, Shaheed Benazir Bhutto University, Sheringal, Pakistan

3 School of Computer Science and Engineering, University of Electronic Science and Technology of China (UESTC), Chengdu 611731, China

4 Department of Software, Sejong University, Seoul 143-747, Republic of Korea

5 Department of Computing, Abasyn University, Peshawar, Pakistan 